TTP97-35

NTZ-24/97

hep-ph/9709352

September 1997

\title{
Lepton pair production by a high energy photon in a strong electromagnetic field
}

\author{
DMITRI IVANOV \\ Naturwissenschaftlich-Theoretisches Zentrum und Institut für Theoretische Physik, \\ Universität Leipzig, Augustusplatz 10, \\ D-04109 Leipzig, Germany \\ and \\ Institut for Mathematics, 630090 Novosibirsk, Russia \\ e-mail: Dmitri.Ivanov@itp.uni-leipzig.de \\ KIRILl MELNiKOV \\ Institut für Theoretische Teilchenphysik \\ Universität Karlsruhe \\ D-76128 Karlsruhe, Germany \\ e-mail:melnikov@ttpux2.physik.uni-karlsruhe.de
}

\begin{abstract}
Using impact-factor representation, we consider the lepton pair production by an incident high-energy photon in a strong electromagnetic field of a nucleus. By summing leading terms of perturbation series, we obtain a simple formula for the amplitude, valid to all orders in $\mathcal{O}(\alpha Z)$ and arbitrary field of the nucleus. Using these results, we derive, in a simple manner, the results for the lepton pair production by a virtual incident photon in a Coulomb field. For real incident photon our results coincide with the known ones. Also, a particular example of a non-Coulomb potential is discussed in some detail.
\end{abstract}




\section{Introduction}

QED processes in the strong Coulomb field attracted considerable attention recently. The understanding of such processes is important for accurate background estimates in heavy ion collisions. Moreover, their analysis is also interesting from pure theoretical point of view. Indeed, the accuracy of perturbative calculations in this case is determined by a parameter $\nu \equiv \alpha Z$, where $\alpha$ is the fine structure constant and $Z$ is the charge of the heavy nucleus. In realistic cases, $\nu \approx 1$ can be achieved and then an exact calculations with respect to this parameter are necessary.

The process of lepton pair production by a high energy $\left(\omega_{\gamma} \gg m\right)$ photon in a strong Coulomb field has been considered in the literature long ago [1] and is even discussed in the text-books [2]. In the standard approach to this problem an exact in $\nu$ result is obtained by using the Coulomb wave functions for the produced charged leptons. Though the final result (especially for the total cross section) is rather simple, intermediate calculations on this way are known to be rather involved.

On the other hand, a powerful way to obtain high energy asymptotic of scattering amplitudes in QED and QCD is based on the impact representation for the scattering amplitude [4, 5]. In this paper we apply this technique for the process of lepton pair production by a high energy photon in a strong electromagnetic field of a nucleus. Using recurrence relations [6], which relate impact factors, describing a conversion of the photon into the lepton pair through an exchange of $\mathrm{N}$ and $\mathrm{N}+1$ photons in the $t$-channel, we sum up the leading terms of the perturbation series in a very efficient way. As a result we obtain a simple formula for the amplitude valid for an arbitrary field of the nucleus.

The representation of the amplitude, which is derived in this paper, is close in its meaning to a famous eikonal representation for a charge-charge scattering. It generalizes the eikonal representation to the case of a dipole-charge scattering and is valid irrespective of an exact form of an interaction potential between leptons and the nucleus. This pleasant feature of our approach permits an easy investigation of the situation, when an interaction potential between the lepton and the nucleus differs from the Coulomb one. This situation emerges, for example, if one considers the nucleus as an extended object and, consequently, describes its interaction with the photon by introducing a form factor $F(k)=\Lambda^{2} /\left(k^{2}+\Lambda^{2}\right)$. Below we consider two limiting cases $\Lambda \ll m$ and $\Lambda \gg m$ in some detail.

For the Coulomb field we obtain the scattering amplitude and the total cross section in the case of a lepton pair production by a virtual photon. As a byproduct of this analysis the known results for the lepton pair production by a real photon are reproduced. We feel, that the use of the representation for the amplitude, derived in this paper, considerably simplifies the calculations in this case. This is especially notable in the case of the total cross section of the reaction $\gamma A \rightarrow l^{+} l^{-} A$.

The paper is organized as follows. In the next section we introduce our notations and give a general discussion of the impact representation. In Section 3 formulas for QED impact 
factors valid to all orders in $\mathcal{O}(\nu)$ are derived and a convenient expression for the amplitude of the process $\gamma^{*} A \rightarrow l^{+} l^{-} A$ is obtained. In Section 4 the total cross section for the reaction $\gamma^{*} A \rightarrow l^{+} l^{-} A$ is derived in a simple manner. Section 5 is devoted to the analysis of the case of a non-Coulomb potential. Finally we present our conclusions.

\section{Basic notations}

A typical Feynman graph which contributes to the leading asymptotic of a lepton pair production by a high energy photon is shown in Fig.1. Our notations are also introduced there.

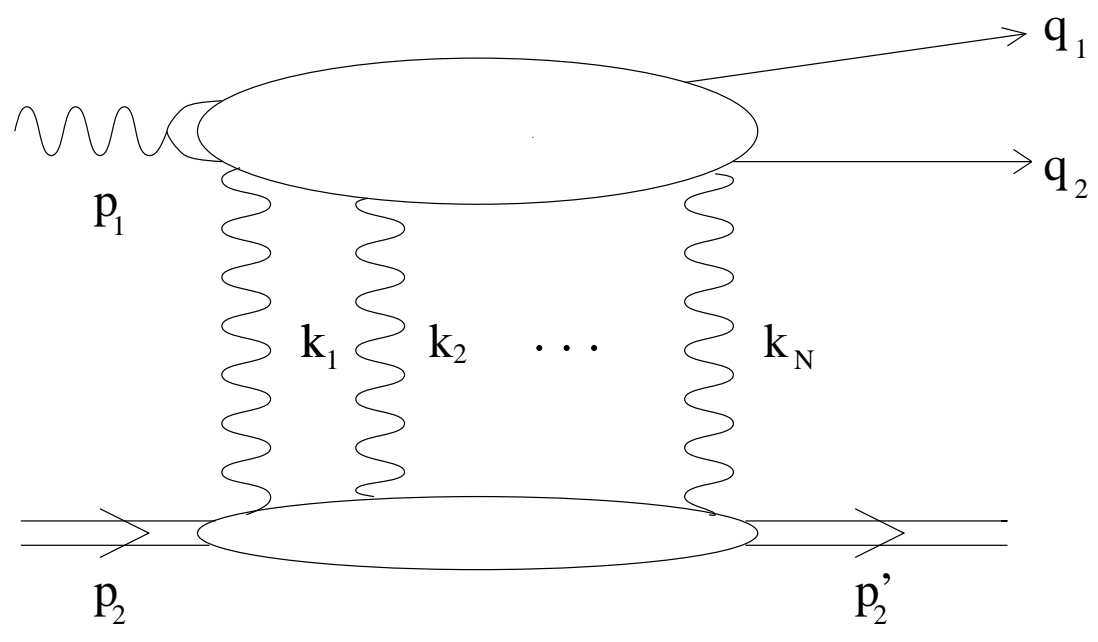

Figure 1: A diagram with $N$-photons, exchanged in the $t$-channel. Diagrams of this type contribute to the leading asymptotic of a lepton pair production by a high energy photon.

The graph in Fig.1 describes an exchange of $\mathrm{N}$ photons in the $t$-channel. The four momenta of the $t$-channel photons are denoted as $k_{i}$. The upper (lower) block in Fig.1 represents an amplitude $A_{\mu_{1} \ldots \mu_{N}}^{N}\left(B_{\mu_{1} \ldots \mu_{N}}^{N}\right)$ with all possible attachments of the $t$-channel photons to the lepton (nuclear) line.

Let us denote the four momentum of the incident photon by $p_{1}$ and its virtuality by $p_{1}^{2}=-Q^{2}$. A transverse polarization vector of the incident photon is $\epsilon$. We denote the four momenta of the produced lepton and antilepton as $q_{1}$ and $q_{2}$, their energies as $\epsilon_{1}$ and $\epsilon_{2}$ and the corresponding Dirac spinors as $u_{1}$ and $u_{2}$. The mass of the lepton is $m$, its charge is $-e$. The antilepton has the same mass and the opposite charge. The charge of the nucleus is $e Z$, 
its four momentum is $p_{2}$.

In what follows, we consider a situation, when the energy of the photon in the rest frame of the nucleus is much larger than the mass of the lepton and the virtuality of the photon:

$$
\omega_{\gamma} \gg m, \sqrt{Q^{2}}
$$

In this case the produced leptons are ultrarelativistic, they fly away in a small open angles $\theta \sim m / \omega_{\gamma}$ with respect to the momentum of the incident photon. The sum of the energies of the produced leptons is equal to the energy of the initial photon with a high accuracy $\sim q^{2} / M_{A}$. Hence, we denote: $\epsilon_{1}=x \omega_{\gamma}, \epsilon_{2}=(1-x) \omega_{\gamma}$.

It will be convenient for us to consider a more general case, when the "nucleus" is a light particle with the mass equal to the mass of the lepton $m$ and the charge $e Z$. The kinematic conditions which ensure the high energy limit of the photoproduction of the lepton pair in this case are

$$
2 p_{1} p_{2}=s \gg m^{2}, Q^{2} .
$$

Note, that in this limit the results for the lepton pair photoproduction coincide with the case of the photoproduction on a heavy nucleus at high energies 1 .

There are two important scales for the square of the transverse (with respect to $p_{1}$ and $p_{2}$ ) part of the transfered momentum $q=q_{1}+q_{2}-p_{1}: \mathbf{q}^{2} \sim m^{2}, Q^{2}$ and $\mathbf{q}^{2} \sim m^{4} / s, Q^{4} / s$. We will consider mainly the first region, where the transverse momentum is not too small:

$$
\mathbf{q}^{2} \sim m^{2}, Q^{2} ; \quad q^{2} \approx-\mathbf{q}^{2} .
$$

For all diagrams of the type of Fig1. with the number of photons in the $t$-channel $N \geq 2$, this region gives the leading contribution to the total cross section. The contribution of the small region $\mathbf{q}^{2} \leq q^{2} \sim m^{4}, Q^{4} / s$ is important only for the diagram with the exchange of one photon in the $t$-channel. Such diagram represents a Born process and therefore can be easily considered separately.

It is convenient to introduce the so-called Sudakov variables. Let us define two almost light-like vectors:

$$
\tilde{p}_{1}^{2}=\tilde{p}_{2}^{2}=0, \tilde{p}_{1}=p_{1}+\frac{Q^{2}}{s} p_{2}, \tilde{p}_{2}=p_{2}-\frac{m^{2}}{s} p_{1}
$$

All Minkowski vectors are decomposed then in $\tilde{p}_{1}, \tilde{p}_{2}$ and two-dimensional vectors, lying in the transverse plane relative to the collision axis:

$$
\begin{aligned}
& k_{i}=\alpha_{i} \tilde{p}_{1}+\beta_{i} \tilde{p}_{2}+\mathbf{k}_{i}, \\
& q_{1}=x_{1} \tilde{p}_{1}+y_{1} \tilde{p}_{2}+\mathbf{q}_{1}, \\
& q_{2}=x_{2} \tilde{p}_{1}+y_{2} \tilde{p}_{2}+\mathbf{q}_{2} .
\end{aligned}
$$

\footnotetext{
${ }^{1}$ This statement is correct for the impact factors but not for the amplitude. The latter receives additional contributions because of the Coulomb phases, which lepton and antilepton acquire in the field of the nucleus. See dedicated discussion after Eq. (28)
} 
From Eq. (2), it follows that $x_{1}, x_{2} \gg y_{1}, y_{2}$. Moreover, it can be shown, that with the accuracy $\sim m^{2} / s$

$$
x_{1}=x, \quad x_{2}=1-x, \quad y_{1}=\frac{m^{2}+\mathbf{q}_{1}^{2}}{x s}, \quad y_{2}=\frac{m^{2}+\mathbf{q}_{2}^{2}}{(1-x) s} .
$$

Our aim is to sum all graphs of the type of Fig.1, with arbitrary number of photons, exchanged in the $t$-channel. One can show, that the leading contribution to the amplitude comes from the loop momenta of the order of $k_{i}^{2} \approx-\mathbf{k}_{i}^{2}$, with $|\mathbf{k}| \sim m, Q,|\mathbf{q}|$.

In this case the impact representation (see [4, 5]) for the amplitude with $\mathrm{N}$ photons, exchanged in the $t$-channel, reads

$$
\mathcal{M}_{N}=\frac{8 \pi^{2} s(-i)^{N-1}}{N !} \int \prod_{i=1}^{N}\left(\frac{\mathrm{d}^{2} \mathbf{k}_{i}}{(2 \pi)^{2} \mathbf{k}_{i}^{2}}\right) \delta\left(\sum_{i=1}^{N} \mathbf{k}_{i}-\mathbf{q}\right) J_{\gamma \rightarrow \overline{l l}}^{N} J_{A}^{N} .
$$

The accuracy of this equation is expected to be of the order of $\sim m^{2} / s, Q^{2} / s, q^{2} / s$. The quantities $J_{\gamma \rightarrow \overline{l l}}^{N}$ and $J_{A}^{N}$ are called impact factors. They describe the upper and lower blocks in Fig.1. These impact factors depend on the transverse momenta of the $t$-channel photons. Explicit formula for the impact factors $J_{\gamma \rightarrow \overline{l l}}^{N}$ and $J_{A}^{N}$ reads:

$$
\begin{aligned}
J_{\gamma \rightarrow \overline{l l}}^{N} & =\int \prod_{i=1}^{N-1}\left(\frac{\mathrm{d}\left(\beta_{i} s\right)}{2 \pi i}\right)(i A)_{\mu_{1} \ldots \mu_{N}} \frac{\tilde{p}_{2}^{\mu_{1}} \ldots \tilde{p}_{2}^{\mu_{N}}}{s^{N}} \\
J_{A}^{N} & =\int \prod_{i=1}^{N-1}\left(\frac{\mathrm{d}\left(\alpha_{i} s\right)}{2 \pi i}\right)(i B)_{\mu_{1} \ldots \mu_{N}} \frac{\tilde{p}_{1}^{\mu_{1}} \ldots \tilde{p}_{1}^{\mu_{N}}}{s^{N}} .
\end{aligned}
$$

The impact factor of the nucleus $J_{A}^{N}$ is a simple quantity, which does not depend on the transverse momenta of the photons in the $t$-channel:

$$
J_{A}^{N}=i(-1)^{N}(e Z)^{N} .
$$

The expressions above are written for the point-like "nucleus". Treating the nucleus as an extended object, one should consider a generalization of Eqs.(5-8). It can be easily shown, that the only change, as compared to the point-like case, consists in substituting the Fourier transform of the electric potential of the nucleus instead of each of the photon propagators $1 / \mathbf{k}_{i}^{2}$ in Eq. (5). An equivalent way to incorporate the effects of the non-Coulomb field is to use Eq. (5) with the Coulomb photon propagator $1 / \mathbf{k}^{2}$, but to consider the impact factor of the nucleus $J_{A}^{N}$ as being dependent on $t$-channel photon momenta. For the static field with the Fourier transform $F(\mathbf{k}) / k^{2}$, this dependence is obtained by multiplying the "Coulomb" impact factor of the nucleus Eq. (8) by the form factors $F\left(\mathbf{k}_{i}\right)$ for each of $N$ exchanged photons. Note that even if the nucleus can not be considered as the source of the static field, the impact representation for the amplitude Eq. (5) is still valid, but the expression for the nuclear impact factor will be more complicated in this case. 
The central quantity for further consideration is the impact factor, which describes a transition of the incident photon to a lepton pair through the exchange of $\mathrm{N}$ photons in the $t$-channel.

Let us start with the simplest case - the exchange of one photon. For transversely polarized photon straightforward calculations give:

$$
J_{\gamma \rightarrow \overline{l l}}^{1}\left(\mathbf{q}_{1}, \mathbf{q}_{2}\right)=i e^{2} \bar{u}_{1}\left[m \hat{\boldsymbol{\epsilon}} S^{1}-2 x\left(\mathbf{T}^{1} \boldsymbol{\epsilon}\right)-\hat{\mathbf{T}}^{1} \hat{\boldsymbol{\epsilon}}\right] \frac{\hat{\tilde{p}}_{2}}{s} u_{2}
$$

In this equation

$$
\begin{aligned}
S^{1} \equiv S^{1}\left(\mathbf{q}_{1}, \mathbf{q}_{2}\right) & =\frac{1}{\mu^{2}+\mathbf{q}_{1}^{2}}-\frac{1}{\mu^{2}+\mathbf{q}_{2}^{2}} \\
\mathbf{T}^{1} \equiv \mathbf{T}^{1}\left(\mathbf{q}_{1}, \mathbf{q}_{2}\right) & =\frac{\mathbf{q}_{1}}{\mu^{2}+\mathbf{q}_{1}^{2}}+\frac{\mathbf{q}_{2}}{\mu^{2}+\mathbf{q}_{2}^{2}}
\end{aligned}
$$

are the scalar and the vector structures, respectively. The quantity $\mu^{2}$ depends on the virtuality of the photon:

$$
\mu^{2}=m^{2}+Q^{2} x(1-x) .
$$

We note, that for the case of a real incident photon Eq.(9) was first derived in Ref. 11].

In case, when the incident virtual photon is longitudinally polarized, the one-photon impact factor reads

$$
J_{\gamma \rightarrow \overline{l l}}^{1}\left(\mathbf{q}_{1}, \mathbf{q}_{2}\right)=-i e^{2} 2 \sqrt{Q^{2}} x(1-x) S^{1}\left(\mathbf{q}_{1}, \mathbf{q}_{2}\right) \bar{u}_{1} \frac{\hat{\tilde{p}}_{2}}{s} u_{2}
$$

In deriving this equation, the polarization vector of the photon was taken to be:

$$
e_{\mu}^{S}=\frac{2 \sqrt{Q^{2}}}{s}\left(\tilde{p}_{2}\right)_{\mu}
$$

Thanks to gauge invariance, the use of this expression is justified.

Impact representation for the amplitude with $\mathrm{N}$ photons, exchanged in the $t$-channel, (see Eq. (5)) and the results for the one-photon impact factors Eqs. (9.12) are our starting points. In the next section we use them to sum the leading contributions of the graphs of the type of Fig1. and to derive a representation for the scattering amplitude, valid to all orders in $\mathcal{O}(\alpha Z)$.

\section{The amplitude to all orders in $\mathcal{O}(\nu)$}

For further calculations we need an expression for the impact factors $J_{\gamma \rightarrow \bar{l} l}^{N}$. It can be shown that the $N$-photon impact factors are similar in their form to the one-photon impact factors: 
a) for transversely polarized incident photon:

$$
J_{\gamma \rightarrow \overline{l l}}^{N}\left(\mathbf{q}_{1}, \mathbf{q}_{2}\right)=i(-e)^{N+1} \bar{u}_{1}\left[m \hat{\boldsymbol{\epsilon}} S^{N}-2 x\left(\mathbf{T}^{N} \boldsymbol{\epsilon}\right)-\hat{\mathbf{T}}^{N} \hat{\boldsymbol{\epsilon}}\right] \frac{\hat{p_{2}}}{s} u_{2} ;
$$

b) for longitudinally polarized incident photon:

$$
J_{\gamma \rightarrow \bar{l}}^{N}\left(\mathbf{q}_{1}, \mathbf{q}_{2}\right)=-i(-e)^{N+1} 2 \sqrt{Q^{2}} x(1-x) \bar{u}_{1} S^{N} \frac{\hat{\hat{p}_{2}}}{s} u_{2} .
$$

The corresponding scalar $S^{N}$ and vector $\mathbf{T}^{N}$ structures depend now on the transverse momenta of the produced leptons and on the transverse momenta of the $t$-channel photons $\left\{\mathbf{k}_{i}\right\}, i=1 \ldots N$. The momentum conservation constraint in this case reads:

$$
\mathbf{q}_{1}+\mathbf{q}_{2}+\sum_{i=1}^{N} \mathbf{k}_{i}=\mathbf{q}
$$

It turns out (see Ref. [6] for more details) that the recurrence relations exist, which relate the scalar and vector structures, calculated for the exchange of $N$ photons in the $t$-channel, and the similar structures, calculated for the exchange of $N-1$ photons. These recurrence relations read:

$$
\begin{aligned}
S^{N}\left(\mathbf{q}_{1}, \mathbf{q}_{2}, \mathbf{k}_{N}\right) & =S^{N-1}\left(\mathbf{q}_{1}, \mathbf{q}_{2}-\mathbf{k}_{N}\right)-S^{N-1}\left(\mathbf{q}_{1}-\mathbf{k}_{N}, \mathbf{q}_{2}\right), \\
\mathbf{T}^{N}\left(\mathbf{q}_{1}, \mathbf{q}_{2}, \mathbf{k}_{N}\right) & =\mathbf{T}^{N-1}\left(\mathbf{q}_{1}, \mathbf{q}_{2}-\mathbf{k}_{N}\right)-\mathbf{T}^{N-1}\left(\mathbf{q}_{1}-\mathbf{k}_{N}, \mathbf{q}_{2}\right) .
\end{aligned}
$$

For the sake of convenience we do not indicate the dependence on $\mathbf{k}_{1} \ldots \mathbf{k}_{N-1}$ in the above formulas.

The above recurrence relations imply, that the impact factors $J_{\gamma \rightarrow \bar{l}}^{N}$ vanish, if any of the $t$-channel photons have zero transverse momentum. This property guarantees the infra-red finiteness of the impact representation for the amplitude. Physically, this is a manifestation of the dipole interaction of soft photons with the pair of leptons. For soft photon with

$$
1 /|\mathbf{k}| \sim 1 / k>>1 / \mu,
$$

the strength of the interaction with the lepton pair is $\sim e k / \mu$. Therefore, when all $N$ photons in the $t$-channel are soft, the scalar and vector structures appear to be strongly suppressed:

$$
S^{N} \sim \mathcal{O}\left(\frac{1}{\mu^{2}}\left(\frac{k}{\mu}\right)^{N}\right), \mathbf{T}^{N} \sim \mathcal{O}\left(\frac{1}{\mu}\left(\frac{k}{\mu}\right)^{N}\right) .
$$

We note also, that the impact factors $J_{\gamma \rightarrow \bar{l} l}^{N}$ are symmetric under the permutations of the momenta of the $t$-channel photons. This property follows from the Bose symmetry and the fact, that all $t$-channel photons have identical polarization (cf. Eq. (6i)). 
The derivation of the recurrence relations will be presented elsewhere [6]. We note, that for the cases $N=2,3$ the impact factors were earlier calculated by direct integration of the Feynman diagrams using Eq. (6) ( see [7, 8]). Using recurrence relations, these results can be easily reproduced.

The knowledge of the recurrence relations is the crucial point for the subsequent analysis. Using them, we sum up the graphs of the type of Fig.1. Below we discuss the resummation for the scalar structure only, the derivation for the vector structure follows along the same lines.

To calculate the amplitude for the lepton pair production we need to calculate the integrals over $t$-channel loop momenta. For this purpose we introduce:

$$
J_{S}^{N}\left(\mathbf{q}_{1}, \mathbf{q}_{2}\right)=\int \prod_{i=1}^{N} \frac{\mathrm{d}^{2} \mathbf{k}_{i}}{k_{i}^{2}} \delta\left(\sum_{i=1}^{N} \mathbf{k}_{i}-\mathbf{q}\right) S^{N} .
$$

Using recurrence relations we find:

$$
J_{S}^{N}\left(\mathbf{q}_{1}, \mathbf{q}_{2}\right)=\int \frac{\mathrm{d}^{2} \mathbf{k}}{\mathbf{k}^{2}}\left(J_{S}^{N-1}\left(\mathbf{q}_{1}, \mathbf{q}_{2}-\mathbf{k}\right)-J_{S}^{N-1}\left(\mathbf{q}_{1}-\mathbf{k}, \mathbf{q}_{2}\right)\right) .
$$

It is advantageous here to write this relation for the Fourier transform of the function $J_{S}^{N}\left(q_{1}, q_{2}\right)$ which is defined through:

$$
J_{S}^{N}\left(x_{1}, x_{2}\right)=\frac{1}{(2 \pi)^{2}} \int e^{i \mathbf{q}_{1} \mathbf{x}_{1}+i \mathbf{q}_{2} \mathbf{x}_{2}} J_{S}^{N}\left(q_{1}, q_{2}\right) \mathrm{d}^{2} \mathbf{q}_{1} \mathrm{~d}^{2} \mathbf{q}_{2}
$$

In this representation, the recurrence relations are greatly simplified:

$$
J_{S}^{N}\left(x_{1}, x_{2}\right)=J_{S}^{N-1}\left(x_{1}, x_{2}\right) 2 \pi \log \left(\frac{x_{1}}{x_{2}}\right) .
$$

The boundary values $J_{S}^{1}\left(x_{1}, x_{2}\right)$ can be derived and read

$$
J_{S}^{1}\left(x_{1}, x_{2}\right)=K_{0}\left(\mu\left|x_{1}-x_{2}\right|\right) \cdot \log \left(\frac{x_{1}}{x_{2}}\right),
$$

where the function $K_{0}(x)$ is the modified Bessel function.

Using then Eq. (23), one easily finds that:

$$
J_{S}^{N}\left(x_{1}, x_{2}\right)=\frac{K_{0}\left(\mu\left|x_{1}-x_{2}\right|\right)}{2 \pi}\left[\pi \log \left(\frac{x_{1}^{2}}{x_{2}^{2}}\right)\right]^{N} .
$$

Final results for the scalar and the vector structures are calculated then by taking the sum over $N$. We define therefore:

$$
J_{S}\left(x_{1}, x_{2}\right)=\sum_{i=1}^{\infty} \frac{(-i \nu / \pi)^{N-1}}{N !} J_{S}^{N}\left(x_{1}, x_{2}\right) .
$$


Using Eq. (25) to perform the summation and taking inverse Fourier transform, we arrive at the final result for the scalar and the vector structures:

$$
\begin{gathered}
J_{S}\left(q_{1}, q_{2}\right)=\frac{i}{(2 \pi)^{2} 2 \nu} \int \mathrm{d}^{2} \mathbf{x}_{1} \mathrm{~d}^{2} \mathbf{x}_{2} e^{-i q_{1} x_{1}-i q_{2} x_{2}} K_{0}\left(\mu\left|\mathbf{x}_{1}-\mathbf{x}_{2}\right|\right)\left[\left(\frac{x_{1}^{2}}{x_{2}^{2}}\right)^{-i \nu}-1\right], \\
\mathbf{J}_{T}\left(q_{1}, q_{2}\right)=\frac{-1}{(2 \pi)^{2} 2 \nu} \int \mathrm{d}^{2} \mathbf{x}_{1} \mathrm{~d}^{2} \mathbf{x}_{2} e^{-i q_{1} x_{1}-i q_{2} x_{2}} \frac{\mu\left(\mathbf{x}_{1}-\mathbf{x}_{2}\right)}{\left|\mathbf{x}_{1}-\mathbf{x}_{2}\right|} K_{1}\left(\mu\left|\mathbf{x}_{1}-\mathbf{x}_{2}\right|\right)\left[\left(\frac{x_{1}^{2}}{x_{2}^{2}}\right)^{-i \nu}-1\right] .
\end{gathered}
$$

For the sake of completeness, we finally present the amplitude of the process $\gamma^{*} A \rightarrow l^{+} l^{-} A$, expressed through the scalar and vector impact factors. We note, however, that the impact representation (Eqs. (5-7)) leaves unaccounted the effect of large longitudinal distances which are responsible for a Coulomb phase of lepton and antilepton in the field of the nucleus. This classical effect has been the subject of a rigorous treatment in the past, its comprehensive discussion in QED can be found in Ref. [9].

According to [9], the effect of soft photons is described by an universal exponential factor. Usually, this factor is known to be infra-red divergent. In our case, however, the infra-red safe difference of the Coulomb phases of lepton and antilepton emerges. We note, that this finite answer is sensitive to the way one treats the "heavy nucleus". In fact, one gets two different results, considering the nucleus as infinitely heavy particle (the situation of actual interest for us) or as a particle with the mass equal to lepton masses (the technical trick used in this paper). Therefore, the formulas below apply to the case of infinitely heavy nucleus only. In this case, we can use the results for the exponential factor as given in [9]. It can be checked that the leading contribution to the soft photon exponential factor comes from the region where $s \alpha_{i} \beta_{i} \sim \mathbf{k}_{i}^{2}$. The contribution from this region can therefore be separated from the contribution of the region $s \alpha_{i} \beta_{i} \ll \mathbf{k}_{i}^{2}$ which is responsible for hard diffractive scattering and which is correctly described by impact representation. For this reason, the exponential factor and the rest of the amplitude, described by impact factors, can be considered separately. The result is then given by a simple multiplication.

We find the following expression for the amplitude:

a) for transversely polarized incident photon:

$$
\mathcal{M}=8 \pi e \nu s\left(\frac{x}{1-x}\right)^{-i \nu} \bar{u}\left[m \hat{\boldsymbol{\epsilon}} J_{S}\left(q_{1}, q_{2}\right)-2 x\left(\mathbf{J}_{T}\left(q_{1}, q_{2}\right) \boldsymbol{\epsilon}\right)-\hat{\mathbf{J}}_{T}\left(q_{1}, q_{2}\right) \hat{\boldsymbol{\epsilon}}\right] \frac{\hat{p}_{2}}{s} u .
$$

b) for longitudinally polarized incident photon:

$$
\mathcal{M}=-16 \pi \operatorname{e} \nu\left(\frac{x}{1-x}\right)^{-i \nu} \sqrt{Q^{2}} x(1-x) \bar{u} J_{S}\left(q_{1}, q_{2}\right) \frac{\hat{p}_{2}}{s} u .
$$

The generalization to the case of a non-Coulomb potential is straightforward. Changing the photon propagator $1 / \mathbf{k}^{2} \rightarrow F(\mathbf{k}) / \mathbf{k}^{2}$, where $F(\mathbf{k})$ is the electromagnetic form factor of the 
nucleus, one can repeat the derivation. One finds, that in this case Eqs. (29,30) are still valid, if the vector and scalar structures are modified:

$$
\begin{gathered}
J_{S}\left(q_{1}, q_{2}\right)=\frac{i}{(2 \pi)^{2} 2 \nu} \int \mathrm{d}^{2} \mathbf{x}_{1} \mathrm{~d}^{2} \mathbf{x}_{2} e^{-i q_{1} x_{1}-i q_{2} x_{2}} K_{0}\left(\mu\left|\mathbf{x}_{1}-\mathbf{x}_{2}\right|\right)\left[e^{-i \nu \varphi\left(\mathbf{x}_{1}, \mathbf{x}_{2}\right)}-1\right] \\
\mathbf{J}_{T}\left(q_{1}, q_{2}\right)=\frac{-1}{(2 \pi)^{2} 2 \nu} \int \mathrm{d}^{2} \mathbf{x}_{1} \mathrm{~d}^{2} \mathbf{x}_{2} e^{-i q_{1} x_{1}-i q_{2} x_{2}} \frac{\mu\left(\mathbf{x}_{1}-\mathbf{x}_{2}\right)}{\left|\mathbf{x}_{1}-\mathbf{x}_{2}\right|} K_{1}\left(\mu\left|\mathbf{x}_{1}-\mathbf{x}_{2}\right|\right)\left[e^{-i \nu \varphi\left(\mathbf{x}_{1}, \mathbf{x}_{2}\right)}-1\right] .
\end{gathered}
$$

where

$$
\varphi\left(\mathbf{x}_{1}, \mathbf{x}_{2}\right)=\frac{1}{\pi} \int \frac{\mathrm{d}^{2} \mathbf{k}}{k^{2}} F(\mathbf{k})\left(e^{i \mathbf{k} \mathbf{x}_{2}}-e^{i \mathbf{k} \mathbf{x}_{1}}\right) .
$$

For the Coulomb potential $F(\mathbf{k})=1$. Then

$$
\varphi\left(\mathbf{x}_{1}, \mathbf{x}_{2}\right)=\varphi_{C}\left(x_{1}, x_{2}\right)=\log \frac{x_{1}^{2}}{x_{2}^{2}}
$$

and Eqs. (27,28) are reproduced.

A few words about helicity amplitudes are in order here. Using the explicit form for the Dirac spinors in Eq. (29), one can show that the parts of the amplitude which are proportional to the scalar and vector structures, correspond to the helicity amplitudes with total helicity of the pair \pm 1 for $J_{S}$ and 0 for $\mathbf{J}_{T}$. In the impact parameter representation, helicity coincides with the projection of a spin on the collision axis. Therefore, in the case of a spherically symmetric potential, $J_{S}\left(\mathbf{x}_{1}, \mathbf{x}_{2}\right)$ and $\mathbf{J}_{T}\left(\mathbf{x}_{1}, \mathbf{x}_{2}\right)$ must correspond to the eigenfunctions of the projection of the total angular momentum on the collision axis with the eigenvalues 0 and \pm 1 , respectively. The correctness of this statement can be seen directly from Eqs. (31, 32).

\section{Coulomb potential}

\subsection{The amplitude}

The formulas presented in the previous section provide a basis for further calculations. In what follows, we consider the scalar structure $J_{S}$ only. The calculation for the vector structure is easily performed along the same lines.

We start with substituting the Fourier transform for the Bessel function $K_{0}\left(\mu\left|x_{1}-x_{2}\right|\right)$ in to Eq. (27). Integrating over $x_{1}$ and $x_{2}$, we arrive at the following expression for $J_{S}$ :

$$
J_{S}\left(q_{1}, q_{2}\right)=\frac{-2 i \nu}{(2 \pi)} \int \frac{\mathrm{d}^{2} \mathbf{k}}{\left(\mathbf{k}^{2}+\mu^{2}\right)\left(\left(\mathbf{k}-\mathbf{q}_{1}\right)^{2}\right)^{1-i \nu}\left(\left(\mathbf{k}+\mathbf{q}_{2}\right)\right)^{1+i \nu}} .
$$

Further integrations can be easily performed using powerful methods developed for calculating one loop Feynman integrals. Introducing Feynman parameters and doing the projective 
transformation [3], we rewrite $J_{S}$ as:

$$
J^{S}\left(q_{1}, q_{2}\right)=\frac{i \nu}{|\Gamma(1-i \nu)|^{2}} \frac{1}{\mu^{2} q^{2}}\left(\frac{\xi_{2}}{\xi_{1}}\right)^{1 \nu}\left[J_{0}+\left(\xi_{1}-1\right) J_{1}+\left(\xi_{2}-1\right) J_{2}\right],
$$

where

$$
\begin{aligned}
& J_{0}=\int \frac{\mathrm{d} u_{1} \mathrm{~d} u_{2} \mathrm{~d} u_{3} \delta\left(1-\sum u_{i}\right) u_{1}^{-i \nu} u_{2}^{i \nu}}{\left(u_{3}+\delta u_{1} u_{2}\right)^{2}}, \\
& J_{1}=J_{2}^{*}=\int \frac{\mathrm{d} u_{1} \mathrm{~d} u_{2} \mathrm{~d} u_{3} \delta\left(1-\sum u_{i}\right) u_{1}^{1-i \nu} u_{2}^{i \nu}}{\left(u_{3}+\delta u_{1} u_{2}\right)^{2}},
\end{aligned}
$$

and

$$
\delta=\frac{q^{2}}{\mu^{2}} \xi_{1} \xi_{2}, \quad \xi_{1,2}=\frac{\mu^{2}}{q_{1,2}^{2}+\mu^{2}}
$$

These integrals are rather simple and can be calculated directly. As an example, we quote the result for $J_{0}$ :

$$
J_{0}=\frac{|\Gamma(1-i \nu)|^{4}}{i \nu}\{F(2-i \nu, 1+i \nu ; 2 ; z)-(1+i \nu) \delta F(2-i \nu, 2+i \nu ; 2 ; z)\}
$$

Here $z=1-\delta$ and $F(a, b ; c ; z) \equiv{ }_{2} F_{1}(a, b ; c ; z)$ is the Gauss hypergeometric function.

The hypergeometric functions can be simplified by using Gauss relations and the formula

$$
\begin{aligned}
F(a, b+1 ; c+1 ; z) & =\frac{c}{c-a} F(a, b ; c ; z)+\frac{c(1-z)}{b(a-c)} F^{\prime}(a, b ; c ; z), \\
F^{\prime}(a, b ; c ; z) & =\frac{\mathrm{d}}{\mathrm{d} z} F(a, b ; c ; z) .
\end{aligned}
$$

Finally, we get the following results for the scalar and the vector structures:

$$
\begin{aligned}
& J_{S}\left(q_{1}, q_{2}\right)=\frac{|\Gamma(1-i \nu)|^{2}}{\mu^{2} q^{2}}\left(\frac{\xi_{1}}{\xi_{2}}\right)^{-i \nu}\left\{\left(\xi_{1}-\xi_{2}\right) F(z)-\frac{i \delta}{\nu}\left(\xi_{1}+\xi_{2}-1\right) F^{\prime}(z)\right\}, \\
& \mathbf{J}_{T}\left(q_{1}, q_{2}\right)=\frac{|\Gamma(1-i \nu)|^{2}}{\mu^{2} q^{2}}\left(\frac{\xi_{1}}{\xi_{2}}\right)^{-i \nu}\left\{\left(\xi_{1} \mathbf{q}_{1}+\xi_{2} \mathbf{q}_{2}\right) F(z)-\frac{i \delta}{\nu}\left(\xi_{1} \mathbf{q}_{1}-\xi_{2} \mathbf{q}_{2}\right) F^{\prime}(z)\right\},
\end{aligned}
$$

where the function $F(z)$ reads:

$$
F(z)=F(i \nu,-i \nu ; 1 ; z)
$$

Inserting Eqs. (40,41) into Eqs. (29, 30) one obtains a final expression for the amplitudes of the process $\gamma^{*} A \rightarrow l^{+} l^{-} A$ in the strong Coulomb field. For the lepton pair production by a real $\left(Q^{2}=0\right)$ incident photon, the expression for the amplitude coincides with the known result [2]. 


\subsection{Total cross section}

Using the representation for the amplitude Eq. (29), one derives the result for the differential cross section of the $\gamma^{*} A \rightarrow l^{+} l^{-} A$ :

$$
d \sigma=\frac{2 m r_{e} \nu^{2}}{\pi^{2}}\left[m^{2}\left|J_{S}\right|^{2}+\left|\mathbf{J}_{T}\right|^{2}\left(x^{2}+(1-x)^{2}\right)\right] \mathrm{d} x \mathrm{~d}^{2} \mathbf{q}_{1} \mathrm{~d}^{2} \mathbf{q}_{2}, \quad r_{e}=\frac{\alpha}{m} .
$$

At this point it is convenient to separate a part of the cross section originating from the exchange of one photon $d \sigma_{1}$ and the other part $d \sigma_{2}$, which describes the contribution of the diagrams Fig. 1 with $N \geq 2$ and their interference with the one photon exchange diagram. For this purpose we write:

$$
d \sigma=d \sigma_{1}+d \sigma_{2}
$$

The one-photon exchange cross sections can be calculated without much problems. For transversely polarized incident photon, the result is given by the following equation:

$$
\sigma_{1}^{T}=\frac{4}{3} Z^{2} \alpha r_{e}^{2} \int_{0}^{1} \mathrm{~d} x\left\{\frac{1}{g(x, z)^{2}}+\frac{2\left(x^{2}+(1-x)^{2}\right)}{g(x, z)}\right\}\left\{\log \left(\frac{2 x(1-x) \omega}{m \sqrt{g(x, z)}}\right)-\frac{1}{2}\right\}
$$

where

$$
g(x, z)=1+z x(1-x), \quad z=\frac{Q^{2}}{m^{2}} .
$$

For longitudinally polarized incident photon the cross section reads:

$$
\sigma_{1}^{S}=\frac{4}{3} Z^{2} \alpha r_{e}^{2} \int_{0}^{1} \mathrm{~d} x \frac{4 z x(1-x)}{g(x, z)^{2}}\left\{\log \left(\frac{2 x(1-x) \omega}{m \sqrt{g(x, z)}}\right)-\frac{1}{2}\right\} .
$$

The integration over $x$ can be done in the above formulas, leading to complicated expressions involving dilogarithms. We do not quote these results here.

In the case of real incident photon, the integration over $x$ is easily performed, leading to the formula for $\sigma_{1}$, first obtained by Bethe and Heitler [10]:

$$
\sigma_{1}=\frac{28}{9} r_{e}^{2} Z^{2} \alpha\left(\log \left(\frac{2 \omega}{m}\right)-\frac{109}{42}\right)
$$

A real challenge in the standard calculations is to get an expression for $d \sigma_{2}$. A common way to calculate $\sigma_{2}$ is to use the formulas for the Coulomb wave functions in the ultrarelativistic limit. In essence, this is equivalent to use our expressions for the scalar and vector structures Eqs. (40, 41). This is not, however, the most efficient way. It turns out that for the derivation of the total cross section the expressions for $J_{S}$ and $\mathbf{J}_{T}$, as given by Eqs. (27,28), are more convenient. 
For transversely polarized incident photon, using Eq. (43), one obtains:

$$
\sigma_{2}^{T}=\frac{2 m r_{e} \nu^{2}}{\pi^{2}} \int_{0}^{1} \mathrm{~d} x\left[m^{2} A_{1}+\left(x^{2}+(1-x)^{2}\right) A_{2}\right] .
$$

For longitudinally polarized incident photon the result is

$$
\sigma_{2}^{S}=\frac{2 m r_{e} \nu^{2}}{\pi^{2}} \int_{0}^{1} d x\left(4 Q^{2} x(1-x) A_{1}\right) .
$$

In Eqs. 48, 49) we used:

$$
\begin{aligned}
& A_{1}=\int \mathrm{d}^{2} \mathbf{q}_{1} \mathrm{~d}^{2} \mathbf{q}_{2}\left(\left|J_{S}\right|^{2}-\left|J_{S}^{\text {one-photon }}\right|^{2}\right), \\
& A_{2}=\int \mathrm{d}^{2} \mathbf{q}_{1} \mathrm{~d}^{2} \mathbf{q}_{2}\left(\left|\mathbf{J}_{T}\right|^{2}-\left|\mathbf{J}_{T}^{\text {one-photon }}\right|^{2}\right) .
\end{aligned}
$$

Using the representation for the scalar and vector impact factors given in Eqs. (27,28) and integrating over $\mathbf{q}_{1}$ and $\mathbf{q}_{2}$, we obtain:

$$
\begin{aligned}
& A_{1}=\frac{1}{4 \nu^{2}} \int \mathrm{d}^{2} \mathbf{x}_{1} \mathrm{~d}^{2} \mathbf{x}_{2} K_{0}^{2}\left(\mu\left|x_{1}-x_{2}\right|\right)\left\{\left[\left(\frac{x_{1}^{2}}{x_{2}^{2}}\right)^{i \nu}-1\right]\left[\left(\frac{x_{1}^{2}}{x_{2}^{2}}\right)^{-i \nu}-1\right]-\nu^{2} \log \left(\frac{x_{1}^{2}}{x_{2}^{2}}\right)\right\}, \\
& A_{2}=\frac{\mu^{2}}{4 \nu^{2}} \int \mathrm{d}^{2} \mathbf{x}_{1} \mathrm{~d}^{2} \mathbf{x}_{2} K_{1}^{2}\left(\mu\left|x_{1}-x_{2}\right|\right)\left\{\left[\left(\frac{x_{1}^{2}}{x_{2}^{2}}\right)^{i \nu}-1\right]\left[\left(\frac{x_{1}^{2}}{x_{2}^{2}}\right)^{-i \nu}-1\right]-\nu^{2} \log \left(\frac{x_{1}^{2}}{x_{2}^{2}}\right)\right\} .
\end{aligned}
$$

It is convenient to introduce new variables for further integration: $\mathbf{R}=\left(\mathbf{x}_{1}+\mathbf{x}_{2}\right) / 2, \mathbf{r}=$ $\mathbf{x}_{1}-\mathbf{x}_{2}$ and $\mathbf{n}=\mathbf{r} / r$. Integrations over $\mathbf{R}$ and $\mathbf{r}$ then completely factorize. One gets therefore:

$$
\begin{aligned}
& A_{1}=\frac{\pi}{2 \nu^{2} \mu^{4}}\left(\int \mathrm{d} x x^{3} K_{0}^{2}(x)\right) I_{\nu}, \quad A_{2}=\frac{\pi}{2 \nu^{2} \mu^{2}}\left(\int \mathrm{d} x x^{3} K_{1}^{2}(x)\right) I_{\nu} \\
& I_{\nu}=\int \mathrm{d}^{2} \mathbf{R}\left\{2-\left(\frac{R^{2}}{(\mathbf{R}-\mathbf{n})^{2}}\right)^{i \nu}-\left(\frac{R^{2}}{(\mathbf{R}-\mathbf{n})^{2}}\right)^{-i \nu}-\nu^{2} \log ^{2}\left(\frac{R^{2}}{(\mathbf{R}-\mathbf{n})^{2}}\right)\right\} .
\end{aligned}
$$

The calculation of $I_{\nu}$ can be done especially simple by introducing dimensional regularization to calculate it term by term. We note that in this case the first (constant) term in the integrand of $I_{\nu}$ does not contribute at all.

One gets:

$$
I_{\nu}=-2 \pi \nu^{2}\{\Psi(1-i \nu)+\Psi(1+i \nu)-2 \Psi(1)\}
$$


where $\Psi(z)=\mathrm{d} \Gamma(z) / \mathrm{d} z$.

Using also

$$
\int \mathrm{d} x x^{3}\left(K_{0}^{2}(x), K_{1}^{2}(x)\right)=\left(\frac{1}{3}, \frac{2}{3}\right),
$$

we obtain the result for the $\sigma_{2}^{T}$ :

$$
\sigma_{2}^{T}(z)=\sigma_{2}^{T}(0) G_{T}(z), \quad z=\frac{Q^{2}}{m^{2}}
$$

In this equation

$$
G_{T}(z)=\frac{3}{7} \int_{0}^{1} \mathrm{~d} x \frac{1+2\left(x^{2}+(1-x)^{2}\right) g(x, z)}{g(x, z)^{2}}
$$

and

$$
\sigma_{2}^{T}(0)=-\frac{28}{9} Z^{2} \alpha r_{e}^{2} f(\nu), \quad f(\nu)=\frac{1}{2}[\Psi(1-i \nu)+\Psi(1+i \nu)-2 \Psi(1)] .
$$

¿From the above formulas one easily obtains the result for the total cross section of the lepton pair production induced by a real photon. Indeed, combining $\sigma_{1}$ and $\sigma_{2}^{T}(0)$ together, we obtain the standard result for the total cross section of the reaction $\gamma A \rightarrow l^{+} l^{-} A$ in the Coulomb field:

$$
\sigma=\sigma_{1}+\sigma_{2}=\frac{28}{9} Z^{2} \alpha r_{e}^{2}\left(\log \left(\frac{2 \omega}{m}\right)-\frac{109}{42}-f(\nu)\right)
$$

The result for the longitudinally polarized incident photon reads:

$$
\sigma_{2}^{S}(z)=-\frac{16}{3} Z^{2} \alpha r_{e}^{2} f(\nu) G_{S}(z), \quad G_{S}(z)=z \int_{0}^{1} \mathrm{~d} x \frac{x(1-x)}{g(x, z)^{2}}
$$

\section{An example of a non-Coulomb potential}

A heavy nucleus is an extended object with the inverse radius

$$
\Lambda \sim 30 \mathrm{MeV}
$$

At such scales, the electric field of the nucleus differs substantially from the Coulomb behavior. Note, that the realistic values of $\Lambda$ fall into the interval which can be described by an inequality:

$$
m_{e} \ll \Lambda \ll m_{\mu},
$$

where $m_{\mu}$ and $m_{e}$ are muon and electron masses, respectively. Therefore, for practical

purposes, it is necessary to consider two limiting cases: small or large $\Lambda$ in comparison with 
the masses of the produced fermion. Below these cases are considered in turn. In what follows, we will assume a particular form of the electromagnetic form factor of the nucleus:

$$
F(k)=\frac{\Lambda^{2}}{\Lambda^{2}+k^{2}} .
$$

In this section we also restrict our consideration to the case of a real incident photon. We note, however, that the results for the virtual incident photon require a simple rearrangement of the formulas presented below and thus can be readily obtained if need arises.

\subsection{The case $m \ll \Lambda$}

In this case a perturbation theory in $m / \Lambda$ can be developed. For the realistic case $m=m_{e}$ the non-Coulomb correction $\sim m^{2} / \Lambda^{2}$ is small for the pair production by a real photon. We note, however, that this correction can become important for the photoproduction by a virtual photon, where the expansion parameter is $\mu^{2} / \Lambda^{2}$.

For the particular choice of the form factor Eq. (61), the phase $\varphi$ in Eqs. (31, 32) can be written as

$$
\begin{aligned}
\varphi & =\varphi_{C}-\delta \varphi \\
\varphi_{C}=\frac{1}{\pi} \int \frac{\mathrm{d}^{2} \mathbf{k}}{k^{2}}\left(e^{i \mathbf{k} \mathbf{x}_{2}}-e^{i \mathbf{k} \mathbf{x}_{1}}\right), \quad \delta \varphi & =\frac{1}{\pi} \int \frac{\mathrm{d}^{2} \mathbf{k}}{k^{2}+\Lambda^{2}}\left(e^{i \mathbf{k} \mathbf{x}_{2}}-e^{i \mathbf{k} \mathbf{x}_{1}}\right) .
\end{aligned}
$$

As was shown above, the total cross section is expressed through the squares of the scalar and vector impact factors, integrated over transverse momenta of both leptons:

$$
\begin{aligned}
& A^{S}=\int \mathrm{d}^{2} \mathbf{q}_{1} \mathrm{~d}^{2} \mathbf{q}_{2}\left|J^{S}\left(q_{1}, q_{2}\right)\right|^{2}, \\
& A^{T}=\int \mathrm{d}^{2} \mathbf{q}_{1} \mathrm{~d}^{2} \mathbf{q}_{2}\left|\mathbf{J}^{T}\left(q_{1}, q_{2}\right)\right|^{2} .
\end{aligned}
$$

By $\delta A^{S, T}$ we denote a correction to these quantities caused by a deviation of the interaction potential from the Coulomb one, in the limit $\Lambda \gg m$. To calculate these corrections we note that the $\mathcal{O}\left(m^{2} / \Lambda^{2}\right)$ order effect originates from the configurations in the coordinate space where either $x_{1} \sim \Lambda^{-1}, x_{2} \sim m^{-1}$, or $x_{2} \sim \Lambda^{-1}, x_{1} \sim m^{-1}$, in this case one of the particles flies through the nucleus. Note that for the vector structure $A^{T}$ an additional contribution of the same order exists, which originates from the configuration $\left|x_{2}-x_{1}\right| \sim x_{1} \sim x_{2} \sim \Lambda^{-1}$, when both particles fly through the nucleus.

We get the following results:

$$
\begin{aligned}
& \delta A^{S}=\frac{(2 \pi)^{2}}{2 \nu^{2} m^{2} \Lambda^{2}} \operatorname{Re}\left\{\left(\frac{4 \Lambda^{2}}{m^{2}}\right)^{i \nu} \frac{\Gamma(1+i \nu)^{4}}{\Gamma(2+2 i \nu)} \Phi_{1}(\nu)\right\} \\
& \delta A^{T}=\frac{(2 \pi)^{2}}{2 \nu^{2} \Lambda^{2}} \operatorname{Re}\left\{\frac{(1+i \nu)}{i \nu}\left(\frac{4 \Lambda^{2}}{m^{2}}\right)^{i \nu} \frac{\Gamma(1+i \nu)^{4}}{\Gamma(2+2 i \nu)} \Phi_{1}(\nu)+\Phi_{2}(\nu)\right\},
\end{aligned}
$$




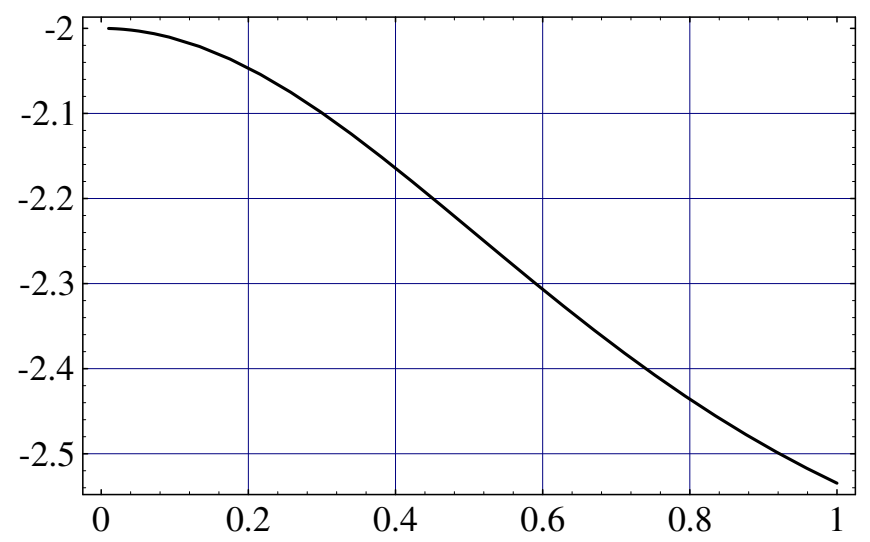

Figure 2: The function $\Phi_{2}(\nu)$ in dependence on $\nu$.

where

$$
\begin{aligned}
& \Phi_{1}(\nu)=\int_{0}^{\infty} \mathrm{d} x x^{1-2 i \nu}\left\{1-e^{-2 i \nu K_{0}(x)}\right\} \\
& \Phi_{2}(\nu)=\frac{1}{(2 \pi)^{2}} \int \frac{\mathrm{d}^{2} \mathbf{x}_{1} \mathrm{~d}^{2} \mathbf{x}_{2}}{\left(\mathbf{x}_{1}-\mathbf{x}_{2}\right)^{2}}\left(\frac{x_{1}^{2}}{x_{2}^{2}}\right)^{i \nu}\left\{1-e^{2 i \nu\left(K_{0}\left(x_{1}\right)-K_{0}\left(x_{2}\right)\right)}\right\} .
\end{aligned}
$$

Though the function $\Phi_{1}(\nu)$ can be integrated numerically without much problems, the representation for the function $\Phi_{2}(\nu)$, presented above, is not well suited for this purpose. Moreover, formally, the expression for $\Phi_{2}(\nu)$, as presented above, is not well-defined and should be understood within analytical regularization. An integral representation for $\Phi_{2}(\nu)$, which respects this property and is suitable for numerical integration, is derived in the Appendix. The plot of the function $\Phi_{2}(\nu)$ is presented in Fig. 2.

Using Eq. (48), we arrive at the $\mathcal{O}\left(\mathrm{m}^{2} / \Lambda^{2}\right)$ correction to the total cross section of $\gamma A \rightarrow$ $l^{+} l^{-} A$ :

$$
\delta \sigma=Z^{2} \alpha r_{e}^{2} \frac{8 m^{2}}{3 \Lambda^{2}} \frac{1}{\nu^{2}} \operatorname{Re}\left\{\left(\frac{2+5 i \nu}{2 i \nu}\right)\left(\frac{4 \Lambda^{2}}{m^{2}}\right)^{i \nu} \frac{\Gamma(1+i \nu)^{2}}{\Gamma(2+2 i \nu)} \Phi_{1}(\nu)+\Phi_{2}(\nu)\right\} .
$$

Taking the values $\Lambda=30 \mathrm{MeV}$ and $m=0.5 \mathrm{MeV}$, we estimate the size of the correction to the case of pure Coulomb potential. Taking, for instance, $\omega_{\gamma}=1 \mathrm{Gev}$, we find that the relative correction does not exceed the -0.3 percent level for all values of $\nu$. Moreover, the $\delta \sigma$ decreases more rapidly with the increase in $\nu$, than the Coulomb cross section does. It is nearly zero for $\nu \sim 1$. For this reason, for larger values of $\nu$, the deviation from Coulomb cross section is even less important. Hence, we conclude that for the electron-positron pair production in the field of a heavy nucleus, one can safely consider the nucleus as a point-like 
source of the Coulomb field. The accuracy of such approximation appears to be extremely good.

It should be stressed however, that the reason for the smallness of the correction is mainly related to the small ratio $m_{e} / \Lambda \sim 10^{-2}$. For the virtual incident photon, the real expansion parameter $\mu^{2} / \Lambda^{2} \sim Q^{2} / \Lambda^{2}$ can be not so small. Simple estimates show that for the virtuality of the incident photon $Q^{2} \sim 10 \mathrm{MeV}$, the effects related to the deviation of the electromagnetic field of the nucleus from the Coulomb behavior can become sizeable.

\subsection{The case $m \gg \Lambda$}

The opposite limit $\Lambda \ll m$ is interesting for the muon pair production in the field of a nucleus. In this case the resummation is not necessary because of dipole interaction of the photon field with the lepton pair. Indeed, the form factor $F(k)$ cuts out all photon momenta $k>\Lambda$. Therefore, the "remaining" long-wave photons with $k \leq \Lambda$, can not resolve a dipole formed by a lepton pair, with typical space separation of the pair components $\sim 1 / m$. For this reason, any photon exchange brings in a suppression factor of $\Lambda / m$.

Hence, for the case $\Lambda \ll m$, it is sufficient to calculate several lowest order diagrams. Though the resummation is not necessary in this case, the representation for the scalar and vector impact factors Eqs. (27,28) appears to be quite useful, in particular for the total cross section.

Without going into any detail of this calculation, we quote the result for the total cross section in the case $\Lambda<<m$ including the first $\mathcal{O}\left(\Lambda^{2} / m^{2}\right)$ correction:

$\sigma=\frac{28}{9} Z^{2} \alpha r_{e}^{2}\left\{\log \left(\frac{2 \omega \Lambda}{m^{2}}\right)-\frac{171}{42}-\frac{3}{35} \frac{\Lambda^{2}}{m^{2}}\left(1+8 c_{1} \nu^{2}\right)\right\}, c_{1}=\int_{0}^{\infty} \frac{\mathrm{d} x}{x^{3}}\left(1-x K_{1}(x)\right)^{4}=0.116$.

Let us make a remark concerning this result. We would like to point out, that Eq. (71) provides a reasonable approximation to the scattering cross section only for very large values of $\omega_{\gamma}$. Indeed, for the values of $\omega_{\gamma}$, less than $\omega_{\text {crit }} \approx 30 \mathrm{~m}^{2} / \Lambda$, the cross section in Eq. (71) is negative. This feature is a consequence of the large negative constant, which accompanies the "large" logarithm 9 and is definitely in accord with the dipole screening, which was emphasized above.

\footnotetext{
${ }^{2}$ We note, that with the logarithmic accuracy Eq. (71) can be easily obtained within equivalent photon approximation.
} 


\section{Conclusions}

In this paper, we have considered a lepton pair production by a high energy photon in the field of the nucleus using impact representation. A simple formula for the scattering amplitude valid to all orders in $Z \alpha$ and arbitrary nuclear potential was derived. This representation is close in its form to the known eikonal representation of scattering amplitudes.

Using this representation, a simple calculation of the total cross section of the reaction $\gamma^{*} A \rightarrow l^{+} l^{-} A$ in the limit $\omega_{\gamma} \gg m, \sqrt{Q^{2}}$ was demonstrated. We hope, that our results for the pair production by a virtual photon will be useful for more accurate calculations of the pair production in lepton-nucleus, proton-nucleus or nucleus-nucleus collisions, than it is usually provided by Equivalent Photon Approximation.

A formula for the amplitude, which is derived in this paper, is valid irrespective of the exact form of the interaction potential between the leptons and the nucleus. For this reason, our approach allows an easy investigation of the case of the non-Coulomb potential. We have considered two limits, of strong and weak deviation of the interaction potential between lepton and nucleus from a Coulomb one and derived the results for the total cross section in these cases. The case of strong deviation is relevant for such processes as $\gamma A \rightarrow \mu^{+} \mu^{-} A$ while the case of weak deviation can be used to describe the $\gamma A \rightarrow e^{+} e^{-} A$ process.

Finally, we would like to emphasize the universality of the obtained results. It is important to realize, that the formulas for the amplitudes (for the scalar and the vector structures), obtained in this paper, play the same role for high-energy dipole-charge scattering as the famous eikonal representation of the scattering amplitudes plays for the charge-charge scattering. In this respect, the results for the impact factors and methods used in this paper, definitely have a broader region of applicability, than the case of pair production which was considered above. In the subsequent paper we are going to demonstrate this by applying our technique to the case of the Delbrück scattering.

\section{Acknowledgments}

We are grateful to R. Kirschner, L. Szymanowski, L.N. Lipatov, V.S. Fadin and T.T. Wu for useful discussions. We are particulary grateful to I.F. Ginzburg who initiated our collaboration on the problem discussed in this paper. This research was supported by BMBF under grants BMBF-057LP91P0 and BMBF-057KA92P and by Graduiertenkolleg "Teilchenphysik" at the University of Karlsruhe. 


\section{Appendix}

In this Appendix a representation for the function $\Phi_{2}(\nu)$, suitable for numerical integration is derived. We start from Eq. (69), where we first integrate over the relative angle of $\mathbf{x}_{1}$ and $\mathbf{x}_{2}$. We then make the change of variables $x_{1} \rightarrow x_{2} r, 0<r<\infty, x_{2} \rightarrow x$ and split the integration region for $r$ into two regions: $0<r<1$ and $1<r<\infty$. Changing the variable $r \rightarrow 1 / r, x \rightarrow x r$ in the second region, we arrive at the following result for $\Phi_{2}(\nu)$ :

$$
\Phi_{2}(\nu)=2 \operatorname{Re}\left\{\int_{0}^{1} \frac{\mathrm{d} r r^{1+2 i \nu}}{1-r^{2}} \int_{0}^{\infty} \mathrm{d} x x\left(1-e^{2 i \nu\left(K_{0}(x r)-K_{0}(x)\right)}\right)\right\} .
$$

It is further convenient to separate the function $\Phi_{2}(\nu)$ into two functions in the following manner:

$$
\Phi_{2}(\nu)=\Phi_{2}^{(0)}(\nu)+\Phi_{2}^{(1)}(\nu)
$$

where

$$
\begin{aligned}
& \Phi_{2}^{(0)}(\nu)=2 \operatorname{Re}\left\{\int_{0}^{1} \mathrm{~d} r r^{1+2 i \nu} \int_{0}^{\infty} \mathrm{d} x x\left(1-e^{2 i \nu\left(K_{0}(x r)-K_{0}(x)\right)}\right)\right\} \\
& \Phi_{2}^{(1)}(\nu)=2 \operatorname{Re}\left\{i \nu \int_{0}^{1} \frac{\mathrm{d} r r^{1+2 i \nu}}{1-r^{2}} \int_{0}^{\infty} \mathrm{d} x x\left[1-e^{2 i \nu\left(K_{0}(x)-K_{0}(x / r)\right)}\right]\right\} .
\end{aligned}
$$

The function $\Phi_{2}^{(1)}$ is already well suited for numerical integration. However, this is not the case for the function $\Phi_{2}^{(0)}(\nu)$. Furthermore, one notes, that for small values of $r$, the function $\Phi_{2}^{(0)}(\nu)$ is proportional to the integral:

$$
\int_{0}^{r_{0}} \mathrm{~d} r r^{-1+2 i \nu}
$$

which is strictly speaking not defined. However, we understand this integral in a sense of analytical regularization, which allows to set the contribution of the low integration boundary in Eq. (76) to zero. By the same token, the following identity should be respected

$$
\int_{0}^{\infty} \mathrm{d} x x^{-1+2 i \nu}=0
$$

within the frame of analytical regularization.

With these definitions, the function $\Phi_{2}^{(0)}(\nu)$ is completely defined and the following result for it can be obtained:

$$
\Phi_{2}^{(0)}(\nu)=-2 \operatorname{Re}\left\{\int_{0}^{\infty} \mathrm{d} x x^{2} K_{1}(x) e^{-2 i \nu K_{0}(x)} \int_{0}^{1} \mathrm{~d} r r^{1+2 i \nu} e^{2 i \nu K_{0}(x r)},\right\}
$$


For the functions $\Phi_{1}^{(0)}$ and $\Phi_{2}^{(0)}$, written in this form, numerical integration can be performed directly, without much problems.

\section{References}

[1] H.A. Bethe and L. Maximon, Phys. Rev. 93 (1954), 788.

[2] L.D. Landau and E.M. Lifschitz, Relativistic Quantum Theory, part 1 (Pergamon, Oxford, 1974).

[3] G. t'Hooft and M. Veltman, Nucl. Phys. B153 (1979), 365.

[4] L.N. Lipatov and G.W. Frolov, Sov. Yad. Fiz. 13, 588 (1971).

[5] H. Cheng and T.T. Wu, Phys. Rev. D1, 3414 (1970).

[6] D.Yu. Ivanov, in preparation.

[7] I.F. Ginzburg, S.L. Panfil and V.G. Serbo, Nucl. Phys. B284 (1987), 685.

[8] I.F. Ginzburg and D.Yu. Ivanov, Nucl. Phys. B388 (1992), 376.

[9] R. Yennie, S. C. Frautschi and H. Suura, Annals of Physics, 13 (1961), 379.

[10] H. A. Bethe and W. Heitler, Proc. R. Soc. A146, (1934), 83.

[11] E. Kuraev, V.G. Serbo and A. Schiller, Zeit. f. Phys C30 (1986), 237. 